\title{
Within-trial effects of stimulus-reward associations
}

Ruth M. Krebs ${ }^{1}$, Jens-Max Hopf ${ }^{2,3}$, Carsten N. Boehler ${ }^{1}$

\author{
${ }^{1}$ Department of Experimental Psychology, Ghent University, Ghent, Belgium \\ ${ }^{2}$ Leibniz Institute for Neurobiology, Magdeburg, Germany \\ ${ }^{3}$ Department of Neurology, University of Magdeburg, Magdeburg, Germany
}

\begin{abstract}
While a globally energizing influence of motivation has long been appreciated in psychological research, a series of more recent studies has described motivational influences on specific cognitive operations ranging from visual attention, to cognitive control, to memory formation. In the majority of these studies, a cue predicts the potential to win money in a subsequent task, thus allowing for modulations of proactive task preparation. Here we describe some recent studies using tasks that communicate reward availability without such cues by directly associating specific task features with reward. Despite abolishing the cue-based preparation phase, these studies show similar performance benefits. Given the clear difference in temporal structure, a central question is how these behavioral effects are brought about, and in particular whether control processes can rapidly be enhanced reactively. We present some evidence in favor of this notion. Although additional influences, for example sensory prioritization of reward-related features, could contribute to the reward-related performance benefits, those benefits seem to strongly rely on enhancements of control processes during task execution. Still, for a better mechanistic understanding of reward benefits in these two principal paradigms (cues vs. no cues), more work is needed that directly compares the underlying processes. We anticipate that reward benefits can be brought about in a very flexible fashion depending on the exact nature of the reward manipulation and task, and that a better understanding of these processes will not only be relevant for basic motivation research, but that it can also be valuable for educational and psychopathological contexts.
\end{abstract}




\section{Introduction}

While a globally energizing influence of (typically reward-induced) motivation on human cognitive and motor functions has long been recognized in psychological research, a series of recent studies has described motivational influences on more specific cognitive operations. Beyond increasing the frequency of a specific action in accordance with the principles of reinforcement learning, the prospect of reward has been shown to improve performance in different domains ranging from visual attention, to cognitive control, to memory formation (e.g., Adcock et al., 2006; Locke and Braver, 2008; Engelmann et al., 2009; Pessoa and Engelmann, 2010; Krebs et al., 2011; Padmala and Pessoa, 2011; Krebs et al., 2012; Schmidt et al., 2012; Chelazzi et al., 2013; Braver et al., 2014). To date, most (but not all) of these studies identified reward effects by using block designs or variants of the monetary-incentive delay (MID) task, in which a cue indicates the prospect of reward for an upcoming task. In this chapter, we will start with a general description of such paradigms for context, but then mostly focus on a more recent line of studies that manipulate reward prospect in a trial-by-trial fashion in the absence of pre-target cues by linking reward prospect directly to features of the target stimuli.

The classic paradigm to study the neural underpinnings of reward effects in humans, the MID task (Knutson et al., 2000; Knutson et al., 2001), has been inspired by research in non-human primates (Schultz et al., 1997; Schultz, 2001). In numerous studies in macaques, these researchers observed increased phasic activity of dopaminergic neurons located in the midbrain when the animals received a primary reward. Critically, after a learning phase, a similar neural response can also be observed for conditioned stimuli that reliably predict such rewards (cues), thus "replacing" the response to the actual reward. This reward-anticipation response is thought to not only reflect reward value, but to simultaneously represent an activating signal that energizes behavioral and mental processes required to obtain the predicted reward (Berridge, 2007; Robbins and Everitt, 2007; Salamone, 2009). In the MID 
task, human participants are typically explicitly informed about the reward contingencies of specific visual cues in advance (mostly bonus money), thereby usually, but not always, omitting the conditioning phase that is typical for animal studies. Analogous to the animal work, it is assumed that reward-predictive cues as well as unexpected rewarding outcomes will trigger activity in the dopaminergic system, while outcomes that are reliably predicted by a cue will not or no longer elicit such responses (Pagnoni et al., 2002; Knutson and Cooper, 2005).

Importantly, dopaminergic responses seem to be capable of altering subsequent neural processing in a number of ways, ranging from a global broadcast signal projected to a widespread set of cortical and subcortical areas, to more local influences on specific neural computations (Braver et al., 2014). As alluded to above, the MID task, that originally involved a simple target-detection task (e.g., Knutson et al., 2000; Knutson et al., 2001), has recently been extended to other research domains, ranging from visual attention (e.g., Engelmann and Pessoa, 2007; Krebs et al., 2012), to various cognitive-control functions (e.g., Padmala and Pessoa, 2011; Schmidt et al., 2012), to memory encoding (e.g., Wittmann et al., 2005; Adcock et al., 2006). Despite substantial differences between these cognitive domains and tasks, the general facilitative effect of reward cues on human performance has proven to be remarkably consistent. This notion has been nicely captured in a recent preview article that titled "Anything you can do, you can do better: neural substrates of incentive-based performance enhancement" (Liljeholm and O'Doherty, 2012). The results of various functional magnetic-resonance imaging (fMRI) studies that employed such cuing paradigms suggest that the beneficial effects of reward seem to rely on modulations of proactive control processes that facilitate the processing of the upcoming target. Hence, it has been proposed that reward acts as a mediator on the respective cognitive functions in a top-down preparatory fashion (Pessoa and Engelmann, 2010; Chelazzi et al., 2013). In other words, the reward information carried by the cue seems to be used in order to prepare an optimal state for successful task performance, which will enable improved target-detection sensitivity (Engelmann and Pessoa, 2007; Engelmann et al., 2009), enhanced suppression of task- 
irrelevant interfering information (Padmala and Pessoa, 2011), or facilitate stimulus encoding into long-term memory (Adcock et al., 2006) - just to name a few processes that seem to be amenable to such reward influences.

The above observations suggest that reward cues largely exert their influence on different cognitive systems in a preparatory manner - before the respective task is actually executed, with actual task execution being superior as a consequence of this rewardtriggered preparation (see also Manelis and Reder, 2013). In addition to fMRI evidence, with its limitation in temporal resolution, there is also evidence from electroencephalography (EEG) studies suggesting that indices of preparatory attention, specifically the contingent negative variation (CNV) and changes in alpha power, are amplified during the cue-target interval of reward compared to no-reward trials, and that the level of preparation indeed relates to subsequent task performance (Schevernels et al., 2014; van den Berg et al., 2014). Moreover, the results of a recent study, in which fMRI and EEG data were acquired simultaneously while participants performed an MID task, suggest that the CNV modulation during reward anticipation is brought about by a combination of subcortical rewardanticipation activity and a top-down regulation of it (Plichta et al., 2013).

While not in the focus of the current chapter, we note that similar beneficial effects have been observed using blocked reward manipulations that likely induce changes in sustained rather than transient preparation, or a combination thereof (e.g., Locke and Braver, 2008; Kouneiher et al., 2009; Jimura et al., 2010; Padmala and Pessoa, 2010; Soutschek et al., 2014). Ignoring differences in experimental design, the results from both reward cuing and blocked reward studies seem to indicate that the beneficial effects of reward prospect on task performance largely arise from proactive top-down control mechanisms that modulate the processing of the succeeding target stimuli. In what follows we will discuss a set of experiments where such proactive control effects are unlikely to impart a differential effect for reward-related trials because it is impossible to predict their occurrence, and hence to prepare for them in a trial-by-trial fashion. 


\section{Within-trial reward manipulations without cues}

Considering the before-mentioned studies, one might come to the conclusion that preparatory proactive control processes are a necessity for the behavioral benefits of reward to occur, especially in tasks that are performed rapidly, which would leave little time to bring about reward-related processing enhancements during task execution. The question of whether reward effects can occur in the absence of such temporally-separated preparation processes may furthermore be ecologically relevant in that real life might not always provide a temporal separation between a moment when reward availability is signaled/registered and when one needs to act on this information. To explore this topic, a recent series of studies has taken a different approach, which excludes a differential influence of global proactive control processes by design. Specifically, information about the availability of reward is linked to features of the target stimulus itself, for example the color, in the absence of a cue that would precede the target in the MID task. Given that such stimulus-reward association (SRA) paradigms don't follow the traditional MID structure (cue-target-feedback), reward benefits in such tasks would likely have to rely on more reactive or even automatic processes compared to MID tasks. Such a distinction could fit well onto a prominent model of cognitive control, the dual mechanisms of control (DMC) framework by Braver and colleagues (Braver et al., 2007; Braver, 2012), which posits that control can flexibly be implemented either proactively or reactively. Although this model usually assumes that reward motivation generally leads to enhanced proactive control (e.g., Jimura et al., 2010), one of the key points of the model is the flexibility with which control can be adapted to different situations. Given this notion, it seems possible that reactive control might also profit from such motivation, in particular in situations in which proactive control cannot easily be used to enhance performance. In the following, we will first introduce two SRA paradigms in some detail, one probing conflict resolution (Krebs et al., 2010) and one probing response inhibition (Boehler et al., 2012). In both paradigms, reward prospect is indicated by the color of the task-relevant stimuli, randomly varied from trial to trial. Afterwards, we will discuss the potential neural 
mechanisms underlying the observed behavioral effects, also with reference to the more common MID manipulations.

Rewarded Stroop task. The first SRA paradigm we will discuss (Krebs et al., 2010) is in essence a regular color-naming Stroop task with manual responses (MacLeod, 1991). In each trial, a word stimulus is presented on the screen in one of four font colors (red, green, blue, yellow) and participants are required to press one out of four buttons to indicate the current font color. Additionally, participants are informed before the experiment that fast and correct responses to two specific font colors (e.g., red and green) can lead to a monetary bonus. As in the regular color-naming Stroop task, the meaning of the word stimulus can be congruent (e.g., the word RED written in red ink), incongruent (e.g., the word GREEN written in red ink) or "neutral" with respect to the font color (e.g., the word BROWN written in red ink), which can be used as a baseline condition. We note that by associating the taskrelevant dimension of the word stimulus (i.e., font color) with reward or no-reward, we created two different types of incongruent word meanings - those that are related to reward and those that are not (e.g., RED vs. BLUE if red is a rewarded font color but blue is not). These incongruent reward-related and reward-unrelated word meanings could occur in both reward and no-reward trials, as could congruent and neutral word meanings. Importantly, word meanings were always irrelevant to the task and never predictive of reward. All trial types were randomly intermixed and occurred with the same probability, which means that participants had no way of knowing whether an upcoming trial would entail the chance for winning a reward or not and thus could not specifically prepare for reward and no-reward trials. Despite this, the behavioral results not only showed facilitation and interference effects commonly observed in the Stroop task, but also a large beneficial effect of reward prospect in the task-relevant dimension (about $100 \mathrm{~ms}$ on average). Interestingly, beyond this facilitative effect of reward in the relevant dimension (font color), interference effects were aggravated if the task-irrelevant dimension of an incongruent trial was related to reward (in the sense that the word referred to a reward color). However, task-relevant reward information seemed to prevent this additional interference. In essence, these results 
demonstrate that within-trial reward information can change the way target stimuli are processed, despite the absence of pre-target cues and the preparatory mechanisms that may be triggered by such cues.

Rewarded Stop-signal task. Inspired by the above findings, we applied the SRA logic to another cognitive-control function, namely response inhibition, i.e., withholding an already initiated response (Boehler et al., 2012). This function is especially interesting in the context of extrinsic motivation as it has been argued that response inhibition is a rather stable feature within individuals (Cohen and Poldrack, 2008), which is derailed in a number of prominent neurological and psychiatric disorders like attention-deficit hyperactivity disorder and substance abuse (Barkley, 1997; Chambers et al., 2009; Ersche et al., 2012). Moreover, response inhibition has to be implemented relatively fast (typically within $250 \mathrm{~ms}$; see below). Response inhibition is often studied using the Stop-signal task (e.g., Verbruggen and Logan, 2008b), in which participants are asked to respond to a target in the majority of trials (e.g., $80 \%$ Go trials), but to withhold their response whenever the target is rapidly followed by a Stop signal (e.g., $20 \%$ Stop trials). This task allows an estimation of how rapidly the initiated "Go-response" can be cancelled by computing the so-called Stop-signal response time (SSRT), which is derived from the stopping success rate in relationship to how fast a given participant is responding in Go trials and the stimulus-onset asynchrony between the Go signal and the Stop signal and usually yields values of around 200 ms (Verbruggen et al., 2013).

In order to investigate whether reward can modulate the ability to inhibit an already initiated response in the absence of any global preparatory control processes, our study provided reward information by the color of the Stop signal in the current paradigm, i.e., only after the Go-response was already triggered (Boehler et al., 2012). In addition to Go trials, the design thus included reward and no-reward Stop trials, which could further be divided in successful and unsuccessful Stop trials based on performance. The crucial questions were whether reward can a) affect response inhibition at all, in particular on such a fast timescale and if so, b) whether stopping would benefit or suffer from these within-trial reward 
associations. The latter outcome would speak for a hard-wired natural mapping between reward and response execution that cannot be easily overruled (e.g., Guitart-Masip et al., 2011; see also Chiu et al., 2014). Despite these alternatives, the results indicate that response inhibition can in fact profit from reward associations, both in terms of a faster SSRT when stopping success was paradigmatically equated across conditions, as well as in terms of stopping success when this was not the case. Both results indicate from different angles that reward information can indeed facilitate the implementation of response inhibition even in the absence of classic preparatory or strategic influences, which have also been generally documented to benefit response inhibition (e.g., Chikazoe et al., 2009). Intriguingly, these effects need to be implemented extremely rapidly (average SSRT around $200 \mathrm{~ms}$ ) and seem to indicate that natural mappings between reward and response execution are either not relevant here, or are somehow overruled.

\section{Possible contributions of reactive control and bottom-up processes}

We conclude from the behavioral results above that the direct association between stimulus features and reward can facilitate performance in the Stroop as well as in the Stop-signal task in the absence of classic global proactive control processes. As a consequence, the question is: how are these within-trial effects are brought about, and how fast can they happen? Given the above documented speed, a key question is whether top-down control mechanisms play a role in bringing about the reward-related benefits, or whether such processes are somehow circumvented.

Before discussing possible contributions of reactive control it is important to clarify what we mean by it. Specifically, we consider processes to reflect enhanced reactive control if they reflect enhancements of operations that take place in the respective tasks, which themselves are usually assumed to reflect reactive control (e.g., Aron, 2011; see also Ridderinkhof et al., 2010; Scherbaum et al., 2011; Braver, 2012). Hence, one could rephrase the question as probing whether reward can rapidly enhance the control functions that are assumed to be involved in the respective cognitive-control function or whether the behavioral benefits are 
brought about in a fashion that does not modulate or even preempt these processes. As such it is referring to active enhancements of the control processes that seem to generally implement the respective tasks (e.g., conflict resolution or response inhibition) rather than for example processes related to a rehashing of the task rules (e.g., Braver et al., 2007). We consider these processes to be enhanced reactively in SRA tasks if they display enhanced activity in reward compared to no-reward trials during task execution. This notion relates to observations that enhanced proactive control usually goes along with diminished activity in cognitive-control areas during task execution, and hence less reactive control (e.g., Paxton et al. 2008; Jimura et al., 2010, Chikazoe et al., 2009, Fan et al., 2007; Luks et al., 2007). This seems to indicate that if reward effects in SRA tasks would largely be driven by some form of proactive control, activity in control areas during task execution should be lower. Yet, one should note that a possible interplay between proactive and reactive processes cannot fully be excluded, as well as possibly independent contributions.

Reactive control within-trials. Based on the above definition, there are indications in support of enhanced within-trial reactive control in the two SRA paradigms presented above. In fMRI versions of both the rewarded Stroop (Krebs et al., 2011) and the rewarded Stopsignal paradigm (Boehler et al., 2014), we observed increased neural activity in the respective task-related cortical networks in reward compared to no-reward trials. Specifically, reward-related Stroop stimuli were associated with enhanced activity in dorsolateral prefrontal and inferior parietal regions, which are commonly implicated in cognitive-control tasks, including conflict processing (Banich et al., 2000; MacDonald et al., 2000; Botvinick et al., 2001; Nee et al., 2007). We performed an analogous analysis for the Stop-signal task. This analysis was further extended by using a conjunction analysis (corresponding to a logical "and") to identify reward modulations that occurred not only in successful (and hence eventually rewarded) Stop trials, but also in unsuccessful (and hence ultimately unrewarded) Stop trials. This approach excludes reward modulations reflecting the subsequent positive evaluation of successful task performance, and should rather target processes that are triggered before the behavioral output. Despite this requirement for modulations to also be 
present in unsuccessful trials, the comparison of reward and no-reward Stop trials revealed a network that included central regions of the inhibition-related network (for reviews see Chambers et al., 2009; Swick et al., 2011), namely a cluster comprising right anterior insula / inferior frontal gyrus as well as a cluster in the dorsal anterior cingulate cortex / presupplementary motor area. This enhanced involvement of critical task-related control areas in both the rewarded Stroop and Stop-signal tasks suggests that reward trials indeed entail enhanced reactive control (see also O'Connor et al., 2014).

Turning back to the relationship between proactive preparation and task execution it is interesting to compare our fMRI Stroop study described above (Krebs et al., 2011) to a similar Stroop study using an MID-like set-up (Padmala and Pessoa, 2011). Specifically, Padmala and Pessoa (2011) employed a picture-word interference task in which a pre-target cue predicted the reward prospect of performance on the upcoming target display (taskrelevant pictures overlaid with task-irrelevant word labels). The behavioral effects of reward were very similar to the ones observed in our rewarded Stroop task, in that the influence of interfering words was reduced in cued reward trials. Moreover, the authors found a joint activation of reward-processing regions and a network of frontal, parietal, and occipital regions implicated in attentional control in response to the cue, which was highly consistent with our fMRI results in the rewarded Stroop task during task execution (Krebs et al., 2011). In the MID context of the Padmala and Pessoa study (2011), this activity pattern is interpreted as proactive control in preparation for the upcoming target in the form of selective attentional filtering of task-irrelevant information. Importantly, this enhanced proactive control went along with attenuated conflict-related activity during actual task execution, hence indeed suggesting that reward-related preparatory processes can alleviate the need for such processes during task execution. This contrasts with another recent study employed an MID version of the Stop-signal task to investigate proactive influences on response inhibition in the context of reward (Rosell-Negre et al., 2014). Here, the prospect of reward benefited Gotrial performance, but also Stop-trial performance in that the SSRT was shorter after reward cues (note that reward cues only indicated that the next trial could yield a reward without 
limiting this possibility to either Go trials or Stop trials) - the latter is analogous to the results of our rewarded Stop-signal paradigm (Boehler et al., 2012). In contrast to the Stroop task by Padmala and Pessoa (2011) discussed above, the study of Rosell-Negre and colleagues (2014) reported enhanced neural activity during response inhibition after reward cues in a network of prefrontal and parietal areas implicated in response inhibition, which resembles the findings of our rewarded Stop-signal task (Boehler et al., 2014). Of note, the neural responses during the cue phase were not explicitly analyzed in the study by Rosell-Negre and colleagues (2014).

This general overlap of neural reward-related effects between MID and SRA studies raises a number of intriguing questions related to the relationship between reward-induced modulations in response to the cue and during actual task execution. In this context, the two aforementioned studies (Padmala \& Pessoa 2011 vs. Rosell-Negre et al. 2014) seem to be somewhat at odds with each other, in the sense that proactive control seems to attenuate the need for control during task performance in the Stroop study (Padmala \& Pessoa 2011), but not in the Stop-signal study (Rosell-Negre et al. 2014). This might relate to the fact that in the conflict task, participants can achieve better performance by enhancing their attentional focus on the task-relevant stimulus dimension, regardless of whether the upcoming task entails a conflict or not. Such focusing is not easily possible in the aforementioned version of the Stop-signal task, because the two potential tasks (stopping and going) would require different preparatory mechanisms, but the cue does not provide any information about the upcoming trial type. Along these lines, it is important to note that activity in areas related to response inhibition can indeed be diminished when participants could proactively prepare to inhibit (Chikazoe et al., 2009). Given that, it is also possible, albeit quite speculative, that reward benefits in a Stop-signal task in which both going and stopping are possibly rewarded are brought about in a fashion that is in fact mostly reactive, even if the general set-up is an MID task.

Relationship to training effects. An important concept to consider is whether the general activity enhancement of task-related networks in reward trials reflects a basic training effect 
that is further enhanced for reward trials. Specifically, one could argue that, although randomly intermixed, reward trials benefit more from practice in terms of a steeper learning curve. Yet, the data does not seem to support this notion. First and foremost, there is no indication for a differential effect of practice in reward trials in the behavioral data. We explicitly tested this in the fMRI Stop-signal paradigm by comparing the reward-related facilitation of SSRT between the first and second half of the experiment (Boehler et al., 2014). While SSRTs were generally shorter in the second half due to practice, this effect was not enhanced for reward trials. We have analyzed the behavioral data of the fMRI Stroop paradigm in the same fashion (Krebs et al., 2011), and found an overall RT facilitation in the second compared to the first half of the experiment $(p<.01)$, but no interaction with the facilitative effect of reward (interaction $\mathrm{p}>.5$; unpublished data).

Although a more fine-grained analysis might be necessary to clearly answer this question, the neural data also seem to speak against a differential practice effect. It has been shown that task-related networks display decreased activity with increased practice in cognitive tasks, including the Stroop task (e.g., Beauchamp et al., 2003; Chen et al., 2013). Enhanced practice effects in reward trials should therefore be reflected in decreased taskrelated activity on average compared to no-reward trials. Similarly, a recent EEG study indicates that training in the Stop-signal task also results in a reduced engagement of the cortical (top-down) response-inhibition network (Manuel et al., 2013), which is the opposite of what we observed for the comparison of reward and no-reward Stop trials. Furthermore, when looking at response-inhibition tasks beyond the standard Stop-signal task, it has been shown that such tasks can (under specific circumstances) lead to the development of an automatic mapping between stimuli and response inhibition (e.g., Verbruggen and Logan, 2008a). This effect, in turn, has been suggested to rely on an alternative route involving parietal areas that circumvents the frontal areas usually involved in response inhibition (Manuel et al., 2010; but see also Lenartowicz et al., 2011; Spierer et al., 2013); again, an effect that our data did not show an indication for as contributing to the reward-related enhancement of response inhibition. Together, it seems that differential practice effects in 
reward versus no-reward trials were not responsible in bringing about the behavioral benefits in our SRA tasks. In both cases, the task-related networks are significantly more active during reward compared to no-reward trials, which seems to indicate an enhanced reactive engagement of the regions that are implicated in the task at hand.

Bottom-up facilitation. That said, there is also evidence for some level of bottom-up prioritization in SRA paradigms, which we will detail in the following section. First, the rewarded Stroop EEG experiment discussed above did also reveal reward-related modulations that preceded the ERP components associated with conflict processing (Krebs et al., 2013). Specifically, we found that reward information was already registered as early as $200 \mathrm{~ms}$ after target onset, reflected in frontal and occipital ERP modulations. These effects are comparable with modulations in response to stimuli that have been associated with a positive outcome (Schacht et al., 2012), as well as to salient emotional stimuli of both negative and positive valence (e.g., Herbert et al., 2006; Stolarova et al., 2006; Kanske, 2012). Moreover, in the visual-search domain, reward prospect has been found to modulate occipital ERP components related to the attentional orienting toward target singletons in a similar time range (Kiss et al., 2009). Together, it seems likely that the early ERP effects observed in the rewarded Stroop task index some sort of bottom-up prioritization of a salient reward-related feature, which could improve behavioral performance in a fashion that may not directly depend on reactive control processes. In addition, it is possible that such prioritization is not (exclusively) reflecting a bottom-up process but could also relate to proactive top-down processes that monitor more strongly for reward-related features in a strategic fashion (see also next section). Whatever the nature of such influences, however, it is important to note that in the SRA designs described above, such a possible sensory prioritization does not seem to alleviate the need for (likely reactive) control processes, in that reward trials still displayed more control-related activity. Yet, it is quite possible that the respective processes interact, such that e.g. an enhanced sensory response could facilitate control processes.Second, we have additional indications for some degree of automaticity with which reward information is processed and affects behavior. One behavioral observation 
supporting this notion is the detrimental effect of task-irrelevant reward information carried by incongruent words in the rewarded Stroop task (Krebs et al., 2010). The most likely explanation for this is that reward-associated colors capture attention (or get prioritized in another fashion), regardless of whether they occur in the task-relevant or task-irrelevant target feature, due to some degree of acquired saliency. If the salient (reward-related) feature is in line with the task goal, and thus with the required behavioral response, this capture leads to response facilitation, while it will disrupt performance if it is incompatible with the task goal (incongruent word meaning). One can moreover approach the question of automaticity by removing reward contingencies from a previously rewarded task, thereby also removing any reward-trial-specific voluntary top-down engagement. We did this in the case of the rewarded Stroop task by adding an extra run with the explicit instruction that no reward is at stake anymore. Despite this instruction, participants were still significantly faster in trials with former reward colors, again speaking in favor of some (residual) enhanced lowlevel saliency/prioritization. We applied a similar logic to the rewarded Stop-signal task and added an extra run in which participants were required to perform a speeded discrimination response to Go stimuli of different colors, including those that were previously used to indicate reward and no-reward Stop trials (Boehler et al., 2012). Like in the extra run of the Stroop task, there was nothing to win in this part of the experiment, which was made explicit to the participants, as well as the fact that there would be no Stop trials. Nevertheless, the results indicate that Go-trial responses were slower for trials that used former reward-related Stop colors compared to former reward-unrelated Stop colors. This suggests that some association between the reward feature and inhibition has been acquired in the main task, which lingers even if the task instructions and the task context (going instead of stopping) change. However, it should be noted that this effect was very small, and that we failed to replicate it (Boehler et al., 2014).

Evidence for the notion that reward can increase sensory saliency comes for a large part from yet another reward regime, namely the biasing of visual attention after reward feedback (Della Libera and Chelazzi, 2006; Hickey et al., 2010; Anderson et al., 2011; Yantis 
et al., 2012; Chelazzi et al., 2013; Chelazzi et al., 2014). The crucial difference to the SRA paradigms presented above is that reward signals modulate attentional selection in an incidental, bottom-up fashion. Specifically, reward is signaled after task execution and is not predictive of reward in the next trial, which excludes the contribution of any proactive and reactive control mechanisms (but see Kristjansson et al., 2010). Nevertheless, these incidental reward signals induce substantial attentional biases, such as performance benefits or costs depending on whether the previous and the current target stimulus share a feature or not. Similar bottom-up effects have also been observed in the conflict-control domain (van Steenbergen et al., 2009; Braem et al., 2012). These studies report a modulation of the behavioral-adaptation effect that is typically observed after incongruent compared to congruent trials by incidental reward feedback. Taken together, it is not possible to exclude contributions of changes in sensory saliency to what we consider enhanced reactive control, but if so, this seems to enhance reactive control rather than circumventing it.

Possible additional proactive effects. We have argued above that the results of the SRA tasks do not reflect the action of global proactive preparatory control as would be typical for MID-like tasks. However, this is not to say that any proactive influences are fully excluded, which theoretically could come in the form of specific preparation for a subset of stimuli/features in an SRA task, or global preparation. Concerning specific preparation, we cannot fully exclude that preparatory effects contribute to our reactive control enhancements, e.g., akin to a prepared reflex that specifies ahead of a trial a rule like "if $X$ happens, do $Y$ " (Verbruggen et al., in press), or top-down attentional biasing for the reward-related stimulus features, which both could be selectively applied to reward-related features. Again, however, if either mechanism would be implemented during the described SRA tasks they do not seem to diminish the necessity for cognitive control during task execution in that activity in controlrelated areas was enhanced rather than reduced in reward trials.

Concerning global preparation, the experiments we have detailed were designed to equate global proactive control levels by making the stimulus sequences unpredictable. Yet, this does not mean that there may not be an additional sustained (proactive) effect brought 
about by the general reward context, which would become visible if one would compare task performance of no-reward trials that are presented in the context of reward trials with trials from a control block that exclusively features no-reward trials. Along such lines, a study on the influence of motivation on working memory has found that when (reward-sensitive) participants switch to a proactive control mode, it will be in fact the intermixed non-reward trials that profit most (Jimura et al., 2010). This latter study used a mixed event-related / block design, which is a very elegant approach for determining global pro-active context effects on no-reward trials. Finally, there are indications that the general context of reward can modulate more incidental aftereffects of behavioral performance - sometimes even to the disadvantage of non-reward trials. In brief, typical performance signatures of a) conflict adaptation (Braem et al., 2012) and b) action-effect binding (Muhle-Karbe and Krebs, 2012) were abolished in no-reward trials when they were intermixed with reward trials. This context effect might arise from a sustained strategic re-distribution of cognitive resources.

The role of dopamine. Although neither fMRI nor EEG data directly reflect neurotransmitter dynamics, we would like to focus on the putative role of dopamine in SRA paradigms, in particular because the SRA task structure leaves substantially less time for any neuromodulatory process to unfold compared to the MID task, in which dopamine is assumed to play a central role. MID tasks were designed to mimic the animal model of the dopaminergic reward-anticipation response in the midbrain (where the cell bodies are located) and (particularly) the ventral striatum (where dopamine is released). Specifically, dopamine neurons increase their phasic activity in response to a cue that reliably predicts a primary reward, and in turn, more dopamine is released in the target regions (Schultz, 2001; Wise, 2004). As this response is highly similar to the one triggered by primary reward itself, it is assumed to reflect the appetitive value of the reward. However, dopamine responses to a cue not only reflect reward value, but are often intermixed with the behavioral activation or mental effort required to obtain the reward (Berridge, 2007; Robbins and Everitt, 2007; Salamone, 2009). Crucially, neural responses in the dopaminergic midbrain can also be elicited in the absence of reward when participants prepare for a high demand versus a low 
demand task (Boehler et al., 2011; see also Krebs et al., 2012), which suggests that the dopaminergic system can be recruited in a top-down fashion. And indeed, there is evidence from functional-connectivity data that lateral prefrontal cortex is the prime input region that explains activity in the dopaminergic midbrain during cue processing in the MID task (Ballard et al., 2011). Together, it seems most likely that cue-locked dopamine responses reflect a mixture of value signals ('I like that') and activation signals ('I will work for it').

In the case of our SRA tasks, the reward feature of the target stimulus is probably to some extent processed like a reward-predicting cue considering that it also acts as a symbol for delayed reward delivery (i.e., bonus money). Indeed, both MID and SRA tasks elicit similar neural responses in the ventral striatum, one of the prime target regions of dopaminergic neurons (e.g., MID: Engelmann et al., 2009; Padmala and Pessoa, 2011; Krebs et al., 2012; SRA: Krebs et al., 2011; Boehler et al., 2014). In keeping with the animal model, these responses could reflect an initial value assessment of the presented cue or target stimulus, which corresponds to the fast phasic response of dopaminergic neurons at about 50 to 100 ms after cue onset (Schultz, 2001). The crucial question that remains to be answered is whether there is enough time for the dopaminergic system to unfold activating, top-down influences in SRA paradigms - in addition to representing the reward value of a given stimulus, with some of the assumed functions presumably requiring some time to unfold (Braver et al., 2007). Intriguingly, the ventral striatum might nevertheless play a role in shaping performance in the SRA version of the Stroop task, in that its activity level was correlated with the behavioral benefit (Krebs et al., 2011). In contrast, in the SRA version of the Stop-signal task, we found that the striatum in all likelihood exclusively signaled performance outcome rather than contributing to shaping it, because it did not display any sensitivity to reward during unsuccessful Stop-trials, which also start off with the prospect of reward but ultimately don't garner it. In the same task, we furthermore found that reward information seemed to impinge on the task-relevant areas via a pregenual anterior cingulate cortex area; a function that in MID-like tasks usually gets assigned to the ventral striatum (Schmidt et al., 2012). This may suggest that reward information can use qualitatively 
different routes to impact behavior, depending on how much time there is for such influences to unfold. As a more general point related to such timing, it might be worth investigating whether the exact mechanism may sometimes not only depend on the sequence of events but also on the speed of progression, wherein a task that by definition involves reactive control might still use mechanisms that fall into a more proactive realm provided that there is sufficient time to implement them. Such general flexibility would again in principal fit the DMC model of Braver and colleagues (Braver et al., 2007; Braver, 2012), which states that the exact nature of control processes will strongly depend on a number of factors pertaining to the task at hand, as well as on differences within and between groups of participants.

\section{Conclusions and outlook}

In the preceding chapter we have described a line of research that is only just unfolding. Specifically, we have reported a number of studies investigating the influence of reward on cognitive-control tasks that breaks with the typical reward-cuing procedure (MID) by associating reward directly to task-relevant stimuli (SRA). While it is evident that reward can nevertheless still have profound effects on behavioral performance, the underlying mechanisms have yet to be fully characterized. In particular, there is evidence that reward associations lead to enhanced activity in task-related control areas, which speaks in favor of the notion that reward associations enhance actual reactive control within a given trial. Yet, there is also evidence for some contribution by more automatic bottom-up processes mostly in the sense of increased saliency/prioritization of reward-related features, which however does not seem to alleviate the need for enhanced activity in control regions during task execution. While these processes mostly seem to act in concert to benefit performance, the bottom-up facilitation of a salient feature that is not compatible with the task goal can disrupt reactive control and ultimately impede performance. This latter observation is clearly in contrast to the solely beneficial effects of preparatory mechanisms triggered by reward cues. An important future extension would be a systematic investigation of the role of dimensional overlap between task-related and reward-related features. In the studies discussed in this 
chapter, this overlap was maximal in the Stroop task, where a subset of target colors was made reward-relevant, so that reward trials were associated to a different behavioral response than no-reward trials. In the Stop-signal task, this overlap was less in that both Stop-stimulus colors required the same response (i.e., response inhibition). Yet, it was still the task-relevant stimulus that also communicated reward availability. Future studies might try to further limit this overlap, which would further emphasize the reactive nature of possible reward-related behavioral benefits. Moreover, while the combined results of different MID and SRA tasks suggest that the balance is tipped more towards proactive preparatory control in the former and more towards reactive control in the latter case, a direct comparison between the two paradigms with closely matched stimuli and task requirements will be critical to further illuminate the relationship and potential interaction between these two control realms.

\section{References}

Adcock RA, Thangavel A, Whitfield-Gabrieli S, Knutson B, Gabrieli JD (2006) Rewardmotivated learning: mesolimbic activation precedes memory formation. Neuron 50:507517.

Anderson BA, Laurent PA, Yantis S (2011) Value-driven attentional capture. Proc Natl Acad Sci U S A 108:10367-10371.

Aron AR (2011) From re-active to proactive and selective control: developing a richer model for stopping inappropriate responses. Biol Psychiatry 69:e55-68.

Ballard IC, Murty VP, Carter RM, Maclnnes JJ, Huettel SA, Adcock RA (2011) Dorsolateral prefrontal cortex drives mesolimbic dopaminergic regions to initiate motivated behavior. $\mathrm{J}$ Neurosci 31:10340-10346.

Banich MT, Milham MP, Atchley R, Cohen NJ, Webb A, Wszalek T, Kramer AF, Liang ZP, Wright A, Shenker J, Magin R (2000) fMRI studies of Stroop tasks reveal unique roles of anterior and posterior brain systems in attentional selection. J Cogn Neurosci 12:9881000. 
Barkley RA (1997) Behavioral inhibition, sustained attention, and executive functions: constructing a unifying theory of ADHD. Psychol Bull 121:65-94.

Beauchamp MH, Dagher A, Aston JA, Doyon J (2003) Dynamic functional changes associated with cognitive skill learning of an adapted version of the Tower of London task. Neuroimage 20:1649-1660.

Berridge KC (2007) The debate over dopamine's role in reward: the case for incentive salience. Psychopharmacology (Berl) 191:391-431.

Boehler CN, Hopf JM, Stoppel CM, Krebs RM (2012) Motivating inhibition - reward prospect speeds up response cancellation. Cognition 125:498-503.

Boehler CN, Schevernels H, Hopf JM, Stoppel CM, Krebs RM (2014) Reward prospect rapidly speeds up response inhibition via reactive control. Cogn Affect Behav Neurosci.

Boehler CN, Hopf JM, Krebs RM, Stoppel CM, Schoenfeld MA, Heinze HJ, Noesselt T (2011) Task-load-dependent activation of dopaminergic midbrain areas in the absence of reward. J Neurosci 31:4955-4961.

Botvinick MM, Braver TS, Barch DM, Carter CS, Cohen JD (2001) Conflict monitoring and cognitive control. Psychol Rev 108:624-652.

Braem S, Verguts T, Roggeman C, Notebaert W (2012) Reward modulates adaptations to conflict. Cognition 125:324-332.

Braver TS (2012) The variable nature of cognitive control: a dual mechanisms framework. Trends Cogn Sci 16:106-113.

Braver TS, Gray JR, Burgess GC (2007) Explaining the Many Varieties of Working Memory Variation: Dual Mechanisms of Cognitive Control. New York: Oxford University Press.

Braver TS et al. (2014) Mechanisms of motivation-cognition interaction: challenges and opportunities. Cogn Affect Behav Neurosci.

Chambers CD, Garavan H, Bellgrove MA (2009) Insights into the neural basis of response inhibition from cognitive and clinical neuroscience. Neurosci Biobehav Rev 33:631-646.

Chelazzi L, Perlato A, Santandrea E, Della Libera C (2013) Rewards teach visual selective attention. Vision Res 85:58-72. 
Chelazzi L, Estocinova J, Calletti R, Lo Gerfo E, Sani I, Della Libera C, Santandrea E (2014) Altering Spatial Priority Maps via Reward-Based Learning. J Neurosci 34:8594-8604.

Chen ZC, Lei X, Ding C, Li H, Chen AT (2013) The neural mechanisms of semantic and response conflicts: An fMRI study of practice-related effects in the Stroop task. Neuroimage 66:577-584.

Chikazoe J, Jimura K, Hirose S, Yamashita K, Miyashita Y, Konishi S (2009) Preparation to inhibit a response complements response inhibition during performance of a stop-signal task. J Neurosci 29:15870-15877.

Chiu YC, Cools R, Aron AR (2014) Opposing Effects of Appetitive and Aversive Cues on Go/No-go Behavior and Motor Excitability. J Cogn Neurosci 26:1851-1860.

Cohen JR, Poldrack RA (2008) Automaticity in motor sequence learning does not impair response inhibition. Psychon Bull Rev 15:108-115.

Della Libera C, Chelazzi L (2006) Visual selective attention and the effects of monetary rewards. Psychol Sci 17:222-227.

Engelmann JB, Pessoa L (2007) Motivation sharpens exogenous spatial attention. Emotion 7:668-674.

Engelmann JB, Damaraju E, Padmala S, Pessoa L (2009) Combined effects of attention and motivation on visual task performance: transient and sustained motivational effects. Front Hum Neurosci 3:4.

Ersche KD, Jones PS, Williams GB, Turton AJ, Robbins TW, Bullmore ET (2012) Abnormal brain structure implicated in stimulant drug addiction. Science 335:601-604.

Fan J, Kolster R, Ghajar J, Suh M, Knight RT, Sarkar R, McCandliss BD (2007) Response anticipation and response conflict: an event-related potential and functional magnetic resonance imaging study. J Neurosci 27:2272-2282.

Guitart-Masip M, Fuentemilla L, Bach DR, Huys QJ, Dayan P, Dolan RJ, Duzel E (2011) Action dominates valence in anticipatory representations in the human striatum and dopaminergic midbrain. J Neurosci 31:7867-7875. 
Herbert C, Kissler J, Junghofer M, Peyk P, Rockstroh B (2006) Processing of emotional adjectives: Evidence from startle EMG and ERPs. Psychophysiology 43:197-206.

Hickey C, Chelazzi L, Theeuwes J (2010) Reward Changes Salience in Human Vision via the Anterior Cingulate. J Neurosci 30:11096-11103.

Jimura K, Locke HS, Braver TS (2010) Prefrontal cortex mediation of cognitive enhancement in rewarding motivational contexts. Proceedings of the National Academy of Sciences of the United States of America 107:8871-8876.

Kanske P (2012) On the influence of emotion on conflict processing. Front Integr Neurosci 6.

Kiss M, Driver J, Eimer M (2009) Reward priority of visual target singletons modulates eventrelated potential signatures of attentional selection. Psychol Sci 20:245-251.

Knutson B, Cooper JC (2005) Functional magnetic resonance imaging of reward prediction. Curr Opin Neurol 18:411-417.

Knutson B, Westdorp A, Kaiser E, Hommer D (2000) FMRI Visualization of Brain Activity during a Monetary Incentive Delay Task. Neurolmage 12:20-27.

Knutson B, Adams CM, Fong GW, Hommer D (2001) Anticipation of increasing monetary reward selectively recruits nucleus accumbens. J Neurosci 21:RC159.

Kouneiher F, Charron S, Koechlin E (2009) Motivation and cognitive control in the human prefrontal cortex. Nat Neurosci 12:939-945.

Krebs RM, Boehler CN, Woldorff MG (2010) The influence of reward associations on conflict processing in the Stroop task. Cognition 117:341-347.

Krebs RM, Boehler CN, Egner T, Woldorff MG (2011) The neural underpinnings of how reward associations can both guide and misguide attention. J Neurosci 31:9752-9759.

Krebs RM, Boehler CN, Appelbaum LG, Woldorff MG (2013) Reward associations reduce behavioral interference by changing the temporal dynamics of conflict processing. PLoS One 8:e53894.

Krebs RM, Boehler CN, Roberts KC, Song AW, Woldorff MG (2012) The involvement of the dopaminergic midbrain and cortico-striatal-thalamic circuits in the integration of reward prospect and attentional task demands. Cereb Cortex 22:607-615. 
Kristjansson A, Sigurjonsdottir O, Driver J (2010) Fortune and reversals of fortune in visual search: Reward contingencies for pop-out targets affect search efficiency and target repetition effects. Atten Percept Psychophys 72:1229-1236.

Lenartowicz A, Verbruggen F, Logan GD, Poldrack RA (2011) Inhibition-related activation in the right inferior frontal gyrus in the absence of inhibitory cues. $\mathrm{J}$ Cogn Neurosci 23:3388-3399.

Liljeholm M, O'Doherty JP (2012) Anything you can do, you can do better: neural substrates of incentive-based performance enhancement. PLoS Biol 10:e1001272.

Locke HS, Braver TS (2008) Motivational influences on cognitive control: behavior, brain activation, and individual differences. Cogn Affect Behav Neurosci 8:99-112.

Luks TL, Simpson GV, Dale CL, Hough MG (2007) Preparatory allocation of attention and adjustments in conflict processing. Neuroimage 35:949-958.MacDonald AW, 3rd, Cohen JD, Stenger VA, Carter CS (2000) Dissociating the role of the dorsolateral prefrontal and anterior cingulate cortex in cognitive control. Science 288:1835-1838.

MacLeod CM (1991) Half a century of research on the Stroop effect: an integrative review. Psychol Bull 109:163-203.

Manelis A, Reder LM (2013) He Who Is Well Prepared Has Half Won The Battle: An fMRI Study of Task Preparation. Cereb Cortex.

Manuel AL, Bernasconi F, Spierer L (2013) Plastic modifications within inhibitory control networks induced by practicing a stop-signal task: an electrical neuroimaging study. Cortex 49:1141-1147.

Manuel AL, Grivel J, Bernasconi F, Murray MM, Spierer L (2010) Brain dynamics underlying training-induced improvement in suppressing inappropriate action. J Neurosci 30:1367013678.

Muhle-Karbe PS, Krebs RM (2012) On the influence of reward on action-effect binding. Front Psychol 3.

Nee DE, Wager TD, Jonides J (2007) Interference resolution: insights from a meta-analysis of neuroimaging tasks. Cogn Affect Behav Neurosci 7:1-17. 
O'Connor DA, Upton DJ, Moore J, Hester R (2014) Motivationally Significant Self-control: Enhanced Action Withholding Involves the Right Inferior Frontal Junction. J Cogn Neurosci:1-12.

Padmala S, Pessoa L (2010) Interactions between cognition and motivation during response inhibition. Neuropsychologia 48:558-565.

Padmala S, Pessoa L (2011) Reward reduces conflict by enhancing attentional control and biasing visual cortical processing. J Cogn Neurosci 23:3419-3432.

Pagnoni G, Zink CF, Montague PR, Berns GS (2002) Activity in human ventral striatum locked to errors of reward prediction. Nat Neurosci 5:97-98.

Paxton JL, Barch DM, Racine CA, Braver TS (2008) Cognitive control, goal maintenance, and prefrontal function in healthy aging. Cereb Cortex 18: 1010-1028.

Pessoa L, Engelmann JB (2010) Embedding reward signals into perception and cognition. Front Neurosci 4.

Plichta MM et al. (2013) Simultaneous EEG and fMRI reveals a causally connected subcortical-cortical network during reward anticipation. J Neurosci 33:14526-14533.

Ridderinkhof KR, Forstmann BU, Wylie SA, van den Wildenberg WP (2010) Neurocognitive mechanisms of action control: resisting the call of the Sirens. Wiley Interdisciplinary Reviews Cognitive Science 2:174-192.

Robbins TW, Everitt BJ (2007) A role for mesencephalic dopamine in activation: commentary on Berridge (2006). Psychopharmacology (Berl) 191:433-437.

Rosell-Negre P, Bustamante JC, Fuentes-Claramonte P, Costumero V, Benabarre S, BarrosLoscertales A (2014) Reward anticipation enhances brain activation during response inhibition. Cogn Affect Behav Neurosci.

Salamone JD (2009) Dopamine, effort, and decision making: theoretical comment on Bardgett et al. (2009). Behav Neurosci 123:463-467.

Schacht A, Adler N, Chen P, Guo T, Sommer W (2012) Association with positive outcome induces early effects in event-related brain potentials. Biol Psychol 89:130-136. 
Scherbaum S, Fischer R, Dshemuchadse M, Goschke T (2011) The dynamics of cognitive control: evidence for within-trial conflict adaptation from frequency-tagged EEG. Psychophysiology 48:591-600.

Schevernels H, Krebs RM, Santens P, Woldorff MG, Boehler CN (2014) Task preparation processes related to reward prediction precede those related to task-difficulty expectation. Neuroimage 84:639-647.

Schmidt L, Lebreton M, Clery-Melin ML, Daunizeau J, Pessiglione M (2012) Neural mechanisms underlying motivation of mental versus physical effort. PLoS Biol 10:e1001266.

Schultz W (2001) Reward signaling by dopamine neurons. Neuroscientist 7:293-302.

Schultz W, Dayan P, Montague PR (1997) A neural substrate of prediction and reward. Science 275:1593-1599.

Soutschek A, Strobach T, Schubert T (2014) Motivational and cognitive determinants of control during conflict processing. Cogn Emot 28:1076-1089.

Spierer L, Chavan CF, Manuel AL (2013) Training-induced behavioral and brain plasticity in inhibitory control. Front Hum Neurosci 7:427.

Stolarova M, Keil A, Moratti S (2006) Modulation of the C1 visual event-related component by conditioned stimuli: evidence for sensory plasticity in early affective perception. Cerebral Cortex 16:876-887.

Swick D, Ashley V, Turken U (2011) Are the neural correlates of stopping and not going identical? Quantitative meta-analysis of two response inhibition tasks. Neuroimage $56: 1655-1665$.

van den Berg B, Krebs RM, Lorist MM, Woldorff MG (2014) Utilization of reward-prospect enhances preparatory attention and reduces stimulus conflict. Cogn Affect Behav Neurosci.

van Steenbergen H, Band GP, Hommel B (2009) Reward counteracts conflict adaptation. Evidence for a role of affect in executive control. Psychol Sci 20:1473-1477. 
Verbruggen F, Logan GD (2008a) Automatic and controlled response inhibition: associative learning in the go/no-go and stop-signal paradigms. Journal of Experimental PsychologyGeneral 137:649-672.

Verbruggen F, Logan GD (2008b) Response inhibition in the stop-signal paradigm. Trends Cogn Sci 12:418-424.

Verbruggen F, Chambers CD, Logan GD (2013) Fictitious inhibitory differences: how skewness and slowing distort the estimation of stopping latencies. Psychol Sci 24:352362.

Verbruggen F, McLaren IPL, Chambers CD (in press) Banishing the control homunculi in studies of action control and behaviour change. Perspectives on Psychological Science.

Wise RA (2004) Dopamine, learning and motivation. Nat Rev Neurosci 5:483-494.

Wittmann BC, Schott BH, Guderian S, Frey JU, Heinze HJ, Duzel E (2005) Reward-related FMRI activation of dopaminergic midbrain is associated with enhanced hippocampusdependent long-term memory formation. Neuron 45:459-467.

Yantis S, Anderson BA, Wampler EK, Laurent PA (2012) Reward and attentional control in visual search. Nebr Symp Motiv 59:91-116. 